\title{
Separación de plata, mercurio y cromo de residuos provenientes de los análisis de demanda química de oxígeno (DQO)
}

\author{
Jorge E. López-Galán*§ \\ * Escuela de Ingeniería Química, Universidad del Valle, Cali, Colombia \\ $\S$ e-mail: jelopez@univalle.edu.co
}

(Recibido: Agosto 26 de 2004 - Aceptado: Noviembre 10 de 2006)

\begin{abstract}
Resumen
Debido a la adición concentrada de sustancias (principalmente $\mathrm{K}_{2} \mathrm{Cr}_{2} \mathrm{O}_{7}, \mathrm{Ag}_{2} \mathrm{SO}_{4}$ y $\mathrm{HgSO}_{4}$ ) usadas en los análisis de demanda química de oxígeno (DQO), al valor económico que tiene la plata, y a los problemas ambientales que el cromo y el mercurio causan, el presente trabajo tiene por objeto separar selectivamente los metales $\mathrm{Cr}, \mathrm{Ag}$ y $\mathrm{Hg}$ de los desechos provenientes de dichos análisis. Para lograr este objetivo, se hicieron varias reflexiones teóricas y se ensayaron varias operaciones unitarias de transferencia de masa, buscando conocer el comportamiento general de algunos reactivos utilizados en cada una de esas operaciones. Con base en los resultados, se considera que se deben separar primero la plata y el mercurio, por precipitación, y después el cromo, por adsorción.
\end{abstract}

Palabras claves: Plata, Mercurio, Cromo, Medio ambiente, Separación selectiva, Operaciones unitarias.

\section{Separation of silver, mercury, and chromium from the wastes resulting from chemical oxygen demand (COD) analyses}

\begin{abstract}
Because of the concentrated addition of substances (mainly $\mathrm{K}_{2} \mathrm{Cr}_{2} \mathrm{O}_{7}, \mathrm{Ag}_{2} \mathrm{SO}_{4}$, and $\mathrm{HgSO}_{4}$ ) used in chemical oxygen demand (COD) analyses, the economic value of silver, and the environmental problems caused by chromium and mercury, the present study is aimed at the selective recovery of $\mathrm{Cr}, \mathrm{Ag}$, and $\mathrm{Hg}$ from the wastes resulting from those analyses. To reach this goal, several mass transfer unit operations were tried out, and several reagents, commonly used in each of such operations, were tested. Among the different procedures used, the precipitation of silver and mercury followed by the adsorption of chromium yielded the best results.
\end{abstract}

Keywords: Silver, Mercury, Chromium, Environment, Selective separation, Unit operations. 


\section{Introducción}

En el método más empleado para los análisis que se hacen para conocer la demanda química de oxígeno (DQO), se utiliza una solución acuosa muy ácida (regularmente dada con el $\mathrm{H}_{2} \mathrm{SO}_{4}$ ), con dicromato de potasio $\left(\mathrm{K}_{2} \mathrm{Cr}_{2} \mathrm{O}_{7}\right)$ para oxidar la materia orgánica, sulfato de plata $\left(\mathrm{Ag}_{2} \mathrm{SO}_{4}\right)$ como catalizador de la oxidación de alcoholes y aminoácidos (colateralmente, también para reducir el efecto negativo de los cloruros) y sulfato de mercurio $\left(\mathrm{HgSO}_{4}\right)$, el cual forma con los cloruros un compuesto muy tóxico de difícil ionización. Una vez finalizado el análisis, se considera que por lo menos el $95 \%$ de la materia orgánica se oxida y que se tienen como productos finales, principalmente: dióxido de carbono $\left(\mathrm{CO}_{2}\right)$ (la mayor parte se difunde en el aire del medio ambiente), residuos de la materia orgánica oxidada (eventualmente puede quedar una pequeña parte sin oxidarse) y la solución acuosa, principalmente con especies de cromo, plata y en menor proporción de mercurio (el que permanece sin reaccionar con los cloruros de la muestra a analizar). En el líquido también se presentan algunos precipitados, principalmente cuando la muestra tiene cloruros (especialmente por la acción de la plata y el mercurio con ellos), además de eventuales compuestos químicamente estables que se forman debido a los enlaces carbonomercurio.

Dada las concentraciones con las que quedan el cromo, la plata y el mercurio en los residuos de los análisis de DQO (máximo del orden de $1.53 \mathrm{~g} / \mathrm{L}$, $3.27 \mathrm{~g} / \mathrm{L}$ y $7.52 \mathrm{~g} / \mathrm{L}$, respectivamente), éstos no se pueden eliminar por las alcantarillas, especialmente por el poder contaminante del cromo y el mercurio, ya que estos valores están muy por encima de lo que recomienda la Organización Mundial de la Salud (los valores deben estar por debajo de $0.1 \mathrm{mg} / \mathrm{L}, 2 \mu \mathrm{g} / \mathrm{L}$ y 25 $\mu \mathrm{g} / \mathrm{L}$, respectivamente en los tres metales) (OMS, 2000). En Colombia, se debe cumplir el decreto 1594 de 1984 (Normativa Ambiental de Aguas) y en México, la norma es la NOM-052-ECOL-1993. Por esta razón, y debido a que la mayor parte de los laboratorios que hacen estos análisis de DQO, poco o nada hacen para solucionar apropiadamente este problema, la presente investigación se orientó hacia la separación de los elementos metálicos adicionados en este proceso de análisis de DQO, para evitar que esos contaminantes lleguen a las alcantarillas, quedando sólo la neutralización de $\mathrm{pH}$ de los líquidos remanentes, para que sean desechados sin problemas ambientales.

Con el fin de lograr los objetivos de la investigación, se estudió el comportamiento teórico de algunas reacciones químicas y se analizaron el tipo de reactivos y las concentraciones respectivas para varias operaciones unitarias de transferencia de masa (extracción líquido-líquido, adsorción y precipitación).

\section{Metodología}

Para obtener los líquidos utilizados en los ensayos, el muestreo se hizo de dos recipientes que contenían alrededor de $18 \mathrm{~L}$ de desechos de los análisis de DQO. Estos líquidos siempre tenían un $\mathrm{pH}$ inferior a 1 .

Los precipitantes y extractantes utilizados en los ensayos de separación selectiva, tenían purezas analíticas, excepto los adsorbentes que fueron de tipo comercial. Las determinaciones analíticas de los metales en los líquidos se hicieron por absorción atómica (se necesitó horno de grafito para el caso del mercurio). Para los precipitados y las muestras sólidas, también se utilizó la absorción atómica y se complementó con análisis de difracción de rayos $\mathrm{X}$.

Para precipitar la plata, se realizaron ensayos con viruta de cinc y con cloruro de sodio $\mathrm{NaCl}$ (en concentraciones mayores a las estequiométricas). Para precipitar el mercurio, se utilizó FeS para generar el ión sulfuro del $\mathrm{H}_{2} \mathrm{~S}$ que precipitó este metal (el exceso de reactivo fue mayor en este caso que en el anterior, por haber un mayor efecto de las reacciones colaterales). Igualmente, se trató de precipitar el mercurio y el hierro, con los iones sulfato del $\mathrm{CaSO}_{4}$, por ser lo más recomendado en la literatura (Cotton et al., 1998), aunque Gould et al. (1984), recomiendan hacerlo con hierro. Como en las precipitaciones quedó una apreciable cantidad de sólidos en suspensión, se hicieron 
algunas pruebas de sedimentación usando floculantes (sulfato de hierro, cal y una mezcla de cloruro férrico con poliacrilamida), variando la concentración desde el orden de los microgramos por litro hasta los gramos por litro, para encontrar el mejor poliectrolito y la concentración más adecuada. Para separar el cromo, se ensayó la precipitación con $\mathrm{Ca}(\mathrm{OH})_{2}$, la extracción líquido líquido con solventes comunes (hexano, dietilenglicol, dibutil éter y xileno) y extractantes disponibles menos comunes (tri-n-octilamina, ácido dietilen triamin penta acético [DTPA] y el ácido di 2-etil-hexil fosfórico) y la adsorción con dos adsorbentes (carbón activado y bentonita), aunque hay otros recomendados en la literatura (Marcus et al., 2002).

Dado que las precipitaciones de plata y mercurio funcionaron bien, los ensayos correspondientes a estas separaciones se centraron en conocer los tiempos, las cantidades de los precipitados y el orden de las precipitaciones. En el caso de la precipitación del cromo, también se observaron los tiempos y la cantidad del precipitado, pero igualmente se analizaron las condiciones operativas de manejo del precipitado, ya que siempre éste fue muy abundante y en varios casos su granulometría era grande. En la extracción líquido-líquido se analizó el tiempo y la capacidad de extracción de los solventes, al igual que en la adsorción con los adsorbentes. Como los ensayos preliminares de adsorción con carbón activado no fueron buenos, el estudio de separación de cromo se centró en ensayos convencionales de adsorción con bentonita para conocer el comportamiento de la temperatura, el tiempo de equilibrio y el $\mathrm{pH}$.

\section{Análisis de resultados}

Se observó que los líquidos residuales almacenados en los laboratorios donde se hacen los análisis de DQO, no son homogéneos porque dependen del origen de las muestras. Por lo tanto, después de un mezclado y el respectivo muestreo al líquido dispuesto, se obtuvo una solución acuosa al 50\% (en volumen) de ácido sulfúrico y una composición promedio de plata, mercurio y cromo de: 1085,823 y 175 ppm, respectivamente. La variación más grande, entre los tres metales de las diferentes muestras originales, la presentó el cromo (entre 158.7 y $207.5 \mathrm{ppm}$ ).
En otros laboratorios latinoamericanos, se han observado variaciones más grandes, no solo del cromo sino de los otros dos elementos. Parece ser que, en el líquido, la plata está como $\mathrm{Ag}^{+}$, el mercurio como $\mathrm{Hg}^{+} \mathrm{y} \mathrm{Hg}^{++}$, y el cromo como $\mathrm{Cr}^{+3} \mathrm{y}$ $\mathrm{Cr}^{+6}$. Estas especies iónicas son variables entre las muestras.

Las precipitaciones de la plata y el mercurio fueron muy cercanas al $99 \%$, utilizando $10 \mathrm{~min}$ de contacto para precipitar la plata (agregando $1.97 \mathrm{~g}$ de sal / L de muestra) y 120 min para el caso del mercurio (agregando $10.4 \mathrm{~g}$ de $\mathrm{FeS} / \mathrm{L}$ de muestra). Las precipitaciones de la plata con viruta de zinc también estuvieron cercanas al $99 \%$, pero los tiempos fueron alrededor de diez veces más largos que los requeridos con el $\mathrm{NaCl}$. La precipitación del mercurio con $\mathrm{CaSO}_{4}$ y limadura de hierro, requirió demasiada cantidad de reactivo y presentó igualmente muchos problemas operativos, por la rápida cristalización de los sulfatos en tamaños muy grandes, lo cual hizo inoperante el manejo de los sólidos, principalmente para realizar la separación de fases sólido-líquido.

El exceso de reactivo requerido fue evidente, un poco más de 3 veces para la plata y 9 veces para el caso del mercurio, lo que supone una buena cantidad de sal en solución y una gran pérdida de $\mathrm{H}_{2} \mathrm{~S}$ y/o la producción de reacciones colaterales con los compuestos resultantes de la oxidación de la materia orgánica, las cuales forman especies iónicas en solución o precipitados. También los precipitados siempre tienen un peso mayor que los establecidos por las estequiometrías de las siguientes reacciones, consideradas como las más viables [definidos con análisis termodinámicos por medio del paquete computacional HSC Chemistry (Outokumpu, 2002)]:

$$
\begin{aligned}
& \mathrm{Ag}_{2} \mathrm{SO}_{4}+2 \mathrm{NaCl} \rightarrow 2 \mathrm{AgCl} \downarrow+\mathrm{Na}_{2} \mathrm{SO}_{4} \\
& \mathrm{FeS}+\mathrm{H}_{2} \mathrm{SO}_{4} \rightarrow \mathrm{H}_{2} \mathrm{~S} \uparrow+\mathrm{FeSO}_{4} \\
& \mathrm{HgSO}_{4}+\mathrm{H}_{2} \mathrm{~S} \rightarrow \mathrm{HgS} \downarrow+\mathrm{H}_{2} \mathrm{SO}_{4} \\
& \mathrm{HgCl}_{2}+\mathrm{H}_{2} \mathrm{~S} \rightarrow \mathrm{HgS} \downarrow+2 \mathrm{Hcl}
\end{aligned}
$$


Esta diferencia de peso es más apreciable para el mercurio $(91.74 \%)$, que para la plata $(1.43 \%)$, lo que significa que, para la plata, la reacción de precipitación es más selectiva. Sin embargo, el análisis de rayos $\mathrm{X}$ mostró que también puede ocurrir la transición de dos fases cristalinas de $\mathrm{Hg}_{3} \mathrm{~S}_{2} \mathrm{Cl}_{2}$.

Para el caso de la precipitación del mercurio, parece que el ácido sulfhídrico hace precipitar apreciablemente (posiblemente como sulfuros), además del mercurio, otros compuestos de la oxidación de la materia orgánica que aún queda en solución y que, por razones dadas anteriormente, pueden llevarse el mercurio. Además, el cloro que no reacciona con la plata puede con el mercurio fácilmente producir también cloruro mercúrico $\left(\mathrm{HgCl}_{2}\right)$ y cloruro mercurioso $\left(\mathrm{Hg}_{2} \mathrm{Cl}_{2}\right)$.

Los análisis de rayos $\mathrm{X}$ muestran que efectivamente hay un predominio de $\mathrm{HgS}$, parte de $\mathrm{FeS}$ sin reaccionar $\mathrm{y}$, posiblemente, $\mathrm{AgS}$ y $\mathrm{AgCrS}_{2}$, cuando aún queda plata en la solución. Sin embargo, según esos análisis, es más evidente que, además del mercurio, el cromo también es precipitado, ya que esos análisis mostraron la significativa presencia de otras sustancias de cromo como $\mathrm{Cr}_{2} \mathrm{O}_{3}, \mathrm{Cr}_{3} \mathrm{O}, \mathrm{Cr} \mathrm{y} / \mathrm{o} \mathrm{HgCr} \mathrm{S}_{4}$ (Marvin, 1956).

Además, en los ensayos de laboratorio se observó que se puede llegar a precipitar hasta el $87 \%$ del cromo cuando se precipita el mercurio por esta vía (los ensayos mostraron precipitaciones que iban desde el 40 hasta el $87 \%$ en peso). Estos resultados aparentemente contradicen la premisa de que la adición del sulfuro ferroso permite no solo precipitar el mercurio sino también reducir el cromo de estado de valencia +6 al estado de valencia +3 , de acuerdo con las siguientes reacciones:
Las anteriores recuperaciones se lograron a temperatura ambiente, lo cual está de acuerdo con la hipótesis de formación del $\mathrm{AgCl}$ dada por la reacción (1), ya que se puede encontrar termodinámicamente que, al modificar la temperatura, la constante de equilibrio no cambia de manera significativa, pues la entalpía de la reacción tiene un valor bajo. Lo mismo sucede con la recuperación del mercurio $(\mathrm{HgS})$. Sin embargo, se observó que la temperatura de la solución se incrementó hasta llegar a los $31^{\circ} \mathrm{C}$. Este hecho pudo principalmente estar determinado por la reducción del cromo +6 a cromo +3 , antes de que el mercurio se precipitara. La reacción de reducción del cromo es exotérmica (aproximadamente $900 \mathrm{~kJ} / \mathrm{mol}$ ); por lo tanto, esto fue lo que seguramente ocasionó el incremento de la temperatura.

La adición del sulfuro ferroso permite no sólo precipitar el mercurio sino reducir el cromo de estado +6 a estado +3 , facilitando su precipitación con sustancias económicas y de fácil adquisición, como son el $\mathrm{NaOH}$, el $\mathrm{Na}_{2} \mathrm{CO}_{3}$ o el $\mathrm{Ca}(\mathrm{OH})_{2}$. Para precipitar más del $95 \%$ de cromo con soda, la cantidad experimental preliminarmente encontrada (basada en observaciones colorimétricas), fue cercana a los $0.5 \mathrm{~g}$ de hidróxido de calcio / $\mathrm{mL}$ de muestra, que es muy grande y, por tanto, no recomendable para un proceso de recuperación rentable de cromo y/o de reducción de desechos. Los precipitados están formados por una gran cantidad de sulfato de calcio dihidratado, por lo cual se considera que el cromo se ocluye en él. Se observó igualmente que el contenido de yeso depende del nivel de DQO. Si éste es menor, el $\mathrm{CaSO}_{4} \cdot 2 \mathrm{H}_{2} \mathrm{O}$ también (el contenido de yeso en el precipitado está aproximadamente entre 73 y $96 \%$ ).

$$
\begin{aligned}
& \mathrm{K}_{2} \mathrm{Cr}_{2} \mathrm{O}_{7}+4 \mathrm{H}_{2} \mathrm{SO}_{4}+3 \mathrm{H}_{2} \mathrm{~S} \rightarrow 3 \mathrm{~S} \downarrow+\mathrm{Cr}_{2}\left(\mathrm{SO}_{4}\right)_{3}+\mathrm{K}_{2} \mathrm{SO}_{4}+7 \mathrm{H}_{2} \mathrm{O} \\
& 2 \mathrm{~K}_{2} \mathrm{CrO}_{4}+5 \mathrm{H}_{2} \mathrm{SO}_{4}+3 \mathrm{H}_{2} \mathrm{~S} \rightarrow 3 \mathrm{~S} \downarrow+\mathrm{Cr}_{2}\left(\mathrm{SO}_{4}\right)_{3}+2 \mathrm{~K}_{2} \mathrm{SO}_{4}+8 \mathrm{H}_{2} \mathrm{O}
\end{aligned}
$$


La cal también mostró un gran poder de precipitación del mercurio, aunque igualmente también las cantidades requeridas son muy altas, lográndose precipitaciones de cromo muy cercanas al $98.5 \%$.

El polielectrolito que mejor funcionó para la sedimentación de las partículas fue una mezcla de coacrilato de sodio y cloruro férrico preparado por el Laboratorio de Síntesis y Mecanismos de Reacciones de Química Orgánica de la Universidad del Valle, utilizando una concentración cercana a $10 \mu \mathrm{g} / \mathrm{L}$.

Aunque De Juan et al. (1998a, 1998b) encontraron buenos resultados para extraer cromo de líquidos acuosos, en nuestros ensayos exploratorios de extracción líquido-líquido, el solvente que mejor resultado dio para separar el cromo, fue la tri-noctilamina disuelta en queroseno, pero como sólo se lograron recuperaciones cercanas al $11 \%$ a los 10 min de tiempo de residencia, en una etapa, se recomienda hacer una investigación mucho más profunda y sistemática para ensayar con más extractantes y definir las mejores condiciones de operación (empezando por definir el tiempo de equilibrio, la capacidad de carga del extractante, la relación de fases, el número de etapas y la concentración del extractante). Sólo así, se sabría si esta operación unitaria es una buena alternativa para recuperar el cromo de esos desechos líquidos.

La adsorción de cromo con carbón activado fue muy pequeña en los ensayos preliminares. Lo máximo que se logró adsorber fue el $12 \%$. Para conocer la capacidad de carga en la adsorción del cromo con bentonita en una etapa (ver Figura 1), se observó que la temperatura prácticamente no influía a $\mathrm{pH}$ cercano a 0 , que el equilibrio se presenta máximo a los $15 \mathrm{~min}$ y que a $\mathrm{pH}$ bajo la adsorción no es buena. Con base en estos resultados y otras pruebas de $\mathrm{pH}$ y temperatura, se encontró la mejor absorción a un $\mathrm{pH}$ de 7.5 y una temperatura de $30^{\circ} \mathrm{C}$. A estas condiciones, para una relación de $35 \mathrm{~g}$ de bentonita / L de solución a un $\mathrm{pH}$ cercano a la neutralidad, la adsorción de cromo fue mayor al $90 \%$, para la mejor capacidad de carga encontrada. Algunos ensayos adicionales mostraron que, cuando no hay plata y mercurio en la solución, las adsorciones mejoran si la operación se realiza a $40{ }^{\circ} \mathrm{C}$, especialmente a condiciones anaeróbicas.

Por efectos colorimétricos presentados en los ensayos realizados, la disolución del cromo en la solución original y en la que quedó después de la

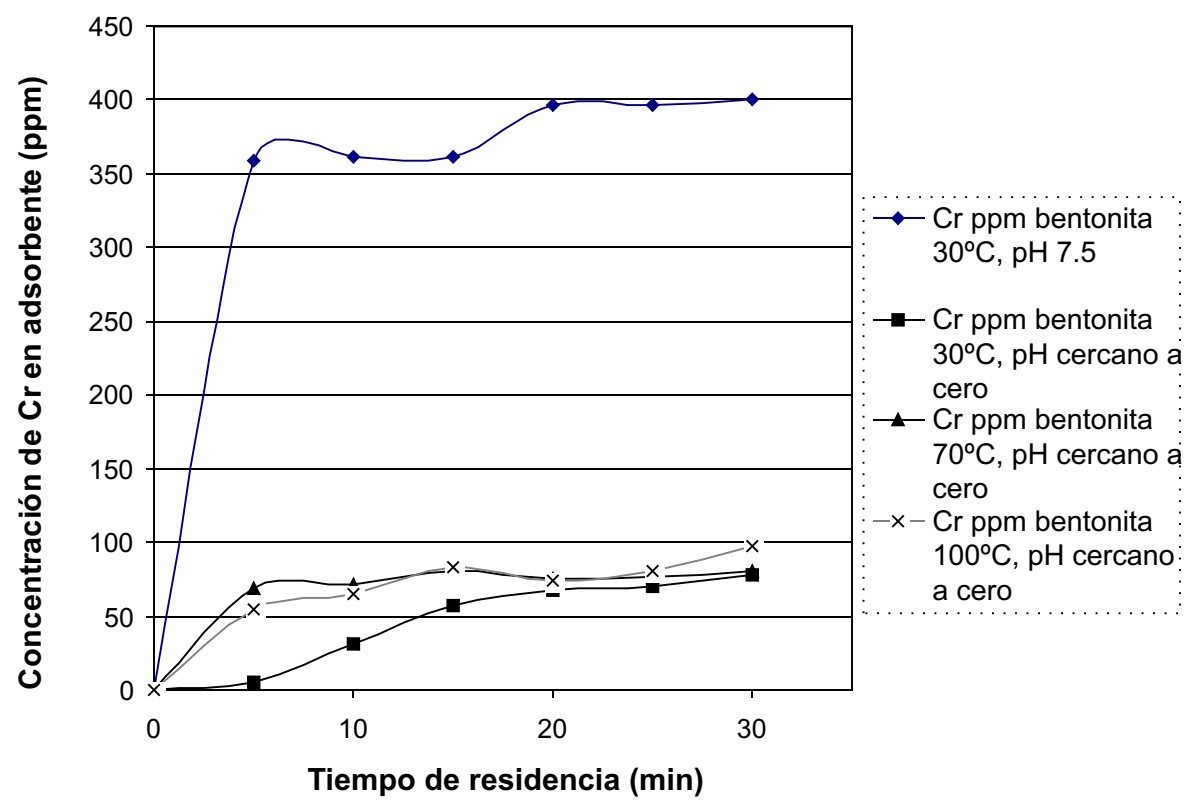

Figura 1. Tendencias del pH y la temperatura con el tiempo. 
precipitación de la plata, presenta un predominio del ión $\left(\mathrm{CrO}_{4}\right)^{-2}$ (color amarillo), luego el hierro y el ácido sulfhídrico lo reducen a $\mathrm{Cr}^{+++}$ (particularmente verde), quedando así después de las precipitaciones. De acuerdo a todas las anteriores reflexiones, se consideró que el proceso más adecuado para tratar los desechos de los análisis de DQO, es el mostrado en la Figura 2:

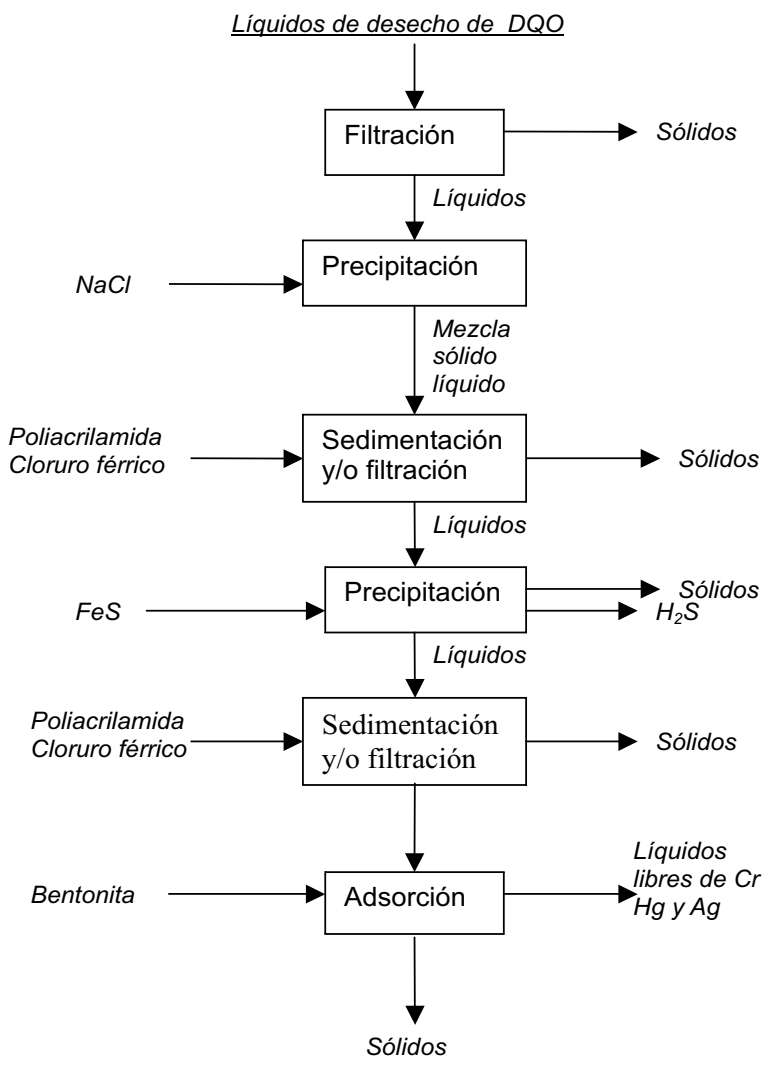

Figura 2. Proceso de separación de metales pesados de los desechos de DQO.

Los líquidos libres de cromo, mercurio y plata, se pueden eliminar después de neutralizarlos (con cal, por ejemplo), dejando así un desecho sin problemas ambientales. Todos los sólidos se pueden reunir y quemar para eliminar los compuestos orgánicos remanentes, y las cenizas se pueden depositar como desechos no peligrosos, especialmente, por tener contenidos muy bajos de cromo y mercurio, además de quedar en condiciones de difícil disolución. Habría antes que estudiar lo que pasa con el mercurio (para evitar que una parte se vaya con los gases producidos en la incineración) y un eventual lavado de los gases, para atrapar el $\mathrm{SO}_{2}$, por ejemplo. Igualmente, se pueden ir acumulando los sólidos obtenidos en cada separación selectiva, para que después de obtener una cantidad apreciable de ellos, se hagan las respectivas fundiciones para obtener la plata, el cromo y el mercurio por separado en forma más concentrada.

\section{Conclusiones}

- Aunque faltaría confirmar con otro tipo de muestras caracterizadas los resultados obtenidos (especialmente, para precisar reacciones colaterales que influyen en la precipitación de los tres metales de estudio), en este trabajo exploratorio de separación selectiva de los metales plata, mercurio y cromo de los desechos resultantes de los análisis de DQO, se encontró que, para que la selectividad sea favorable, la secuencia de separación de los metales en cuestión primero debe ser en el siguiente orden: primero la plata, luego el mercurio y finalmente el cromo. Este orden también está muy relacionado con los comportamientos que esos metales presentaron con cada uno de los métodos y reactivos que se utilizaron, siendo más difícil separar el cromo que el mercurio y este a su vez más difícil que la plata.

- Después de las operaciones de separación selectiva de los elementos químicos en cuestión, quedan tres productos finales concentrados en cada uno de los mismos, que pueden ser utilizados, después de fundirlos, como materias primas de nuevos productos con requerimientos del respectivo metal.

- La precipitación de la plata funciona con rendimientos altos, agregando en un medio agitado $\mathrm{NaCl}$ [utilizando un excedente máximo de 3 veces el estequiométrico, ver reacción (1)], a condiciones ambientales y teniendo un tiempo de contacto de $10 \mathrm{~min}$. Para la precipitación del mercurio, inicialmente el $\mathrm{FeS}$ es bastante apropiado, aunque se deben tener los cuidados respectivos para controlar el $\mathrm{H}_{2} \mathrm{~S}$ que eventualmente se escapa de la solución, estableciendo, por ejemplo, un circuito refrigerado, ojalá cerrado, o lavándolo con agua residual del proceso. Además, hay que tener 
especial cuidado con los cloruros de mercurio que se producen por el exceso de $\mathrm{NaCl}$ utilizado en la precipitación de la plata. En este caso del mercurio, el tiempo de contacto es más largo (alrededor de dos horas) y la adición del precipitante es mayor [cerca de 9 veces el estequiométrico, ver reacciones (2), (3) y (4)]. Los precipitados del mercurio son los que más fases sólidas tienen, ya que posiblemente tienen sulfuros de otros elementos presentes en la materia orgánica inicial. Por lo tanto, posiblemente se presenten dificultades para su refinación.

- La plata debe precipitarse primero que el mercurio, especialmente porque así se puede garantizar que gran parte del mercurio se encuentre como cloruro mercúrico $\mathrm{HgCl}_{2}$, sin interferir en la precipitación selectiva de la plata. Si se desea precipitar al mismo tiempo la plata y el mercurio, lo más indicado es agregar directamente el $\mathrm{H}_{2} \mathrm{~S}$, aunque entonces los problemas de separación de los dos elementos para obtener subproductos son mayores. La reacción de precipitación de la plata, para este caso, da acantita $\left(\mathrm{Ag}_{2} \mathrm{~S}\right)$, de acuerdo con la siguiente reacción:

$$
\mathrm{Ag}_{2} \mathrm{SO}_{4}+\mathrm{H}_{2} \mathrm{~S} \rightarrow \mathrm{Ag}_{2} \mathrm{~S} \downarrow+\mathrm{H}_{2} \mathrm{SO}_{4}
$$

- Aunque el cromo puede quedar en todos los sólidos, a medida que se van realizando las precipitaciones, el cromo +3 se va volviendo más inestable y por lo tanto el cromo tiene cada vez más tendencia a estar soluble. Una buena forma de mantener este ión, es agregando sulfato ferrroso amoniacal $\left[\mathrm{Fe}\left(\mathrm{NH}_{4}\right)_{2}\left(\mathrm{SO}_{4}\right)_{2} \cdot 6 \mathrm{H}_{2} \mathrm{O}\right]$, a pesar de que ésto significaría tener más impurezas.

- El pH es una variable que afecta bastante el comportamiento no sólo del cromo, sino también del mercurio, porque, por ejemplo, la neutralización parcial del ácido sulfúrico con cal o hidróxido, trae como consecuencia la producción de óxido mercúrico $(\mathrm{HgO})$, que es insoluble, y el cloruro de sodio provoca la precipitación de la plata, de acuerdo con las siguientes reacciones:

$$
\begin{aligned}
& \mathrm{HgCl}_{2}+2 \mathrm{NaOH} \rightarrow \mathrm{HgO} \downarrow+2 \mathrm{NaCl}+\mathrm{H}_{2} \\
& \mathrm{Ag}_{2} \mathrm{SO}_{4}+2 \mathrm{NaCl} \rightarrow 2 \mathrm{AgCl} \downarrow+\mathrm{Na}_{2} \mathrm{SO}_{4}
\end{aligned}
$$

Además, el uso de hidróxido produce la formación de precipitado negro de óxido de plata:

$$
\mathrm{Ag}_{2} \mathrm{SO}_{4}+2 \mathrm{NaOH} \rightarrow \mathrm{Ag}_{2} \mathrm{O} \downarrow+\mathrm{Na}_{2} \mathrm{SO}_{4}+\mathrm{H}_{2} \mathrm{O}
$$

- Dado que el pH de los desechos de los análisis de DQO es muy bajo, la cantidad requerida de neutralizante del $\mathrm{pH}$ es muy alta (por el efecto logarítmico), especialmente, si se trata de hacerlo con agua para no involucrar más impurezas en la solución. Por tanto, la neutralización más cómoda resultó ser con la cal.

-Aunque se hicieron muchos intentos para separar el cromo con rendimientos altos, buena selectividad y en forma económica, no se logró tener un método apropiado. Sin embargo, quedaron ciertos fundamentos para optimizar algunos procedimientos que requieren más experimentación y análisis más profundos. Inicialmente, la adsorción con bentonita fue la mejor operación para separar este metal de la solución, faltando aún algunos detalles de optimización.

\section{Recomendaciones}

- Siempre sería mejor prevenir que solucionar los problemas ambientales. En este sentido, dado que entre las sustancias agregadas en el análisis de DQO, la más peligrosa es el mercurio, luego el cromo, después el ácido sulfúrico y por último, la plata, es importante investigar un sustituto de los dos primeros para realizar esos análisis. Por ejemplo, sería interesante investigar un método normalizado de análisis de DQO utilizando ozono, ya que este es un oxidante mucho más fuerte que el dicromato, y se tienen mejores posibilidades para controlar su consumo y sus desechos. Es posible también, que con este oxidante no haya necesidad de poner en digestión la muestra en una solución tan ácida o que se pueda reducir el tiempo y la temperatura de dicha digestión.

Por la gran posibilidad de reacciones y condiciones del proceso, las eficiencias y los reactivos adicionados en las operaciones encontradas como las más adecuadas, dependen en una gran medida del origen de las muestras a las que se les hace los análisis de DQO y de las 
condiciones de dichos análisis (principalmente, el tiempo de contacto, la temperatura y el tipo y cantidad de oxidante utilizado). Por lo tanto, es importante tratar de separar las aguas industriales de las domésticas (lo ideal sería también agruparlas por tipo de industria), para evitar excesos de reactivo en algunos casos y tiempos de contacto largos en otros, con lo cual se podría incluso mejorar las eficiencias, especialmente para el caso del cromo. Además sería importante para cada tipo de agrupamiento hacer caracterizaciones de las aguas y estudios de ajustes de tiempo y cantidad de reactivo, principalmente.

Sería importante investigar un poco más el manejo de los sólidos en la precipitación del mercurio con sulfato de calcio y hierro, encontrando las condiciones más adecuadas de operación $(\mathrm{pH}$, tiempo, cantidad de reactivo y temperatura principalmente), entre otras, para retirar el $\mathrm{Hg}_{2} \mathrm{Cl}_{2}$, que es poco soluble, y que se obtiene de acuerdo con la siguiente reacción:

$$
\mathrm{Fe}+2 \mathrm{HgCl}_{2} \rightarrow \mathrm{FeCl}_{2}+\mathrm{Hg}_{2} \mathrm{Cl}_{2}
$$

Para la adsorción del cromo con bentonita, inicialmente se recomienda realizarlo en un proceso por lotes, a $\mathrm{pH}$ cercano a la neutralidad. Sin embargo, hay que insistir en la extracción líquido-líquido y en la adsorción con polímeros selectivos del cromo, buscando realizar la operación a $\mathrm{pH}$ cercanos al de la muestra líquida original de las determinaciones de DQO.

- Se recomienda investigar la adsorción del cromo utilizando desechos biomásicos como el bagazo y la cáscara de arroz, ya que en varios artículos se han reportado interesantes resultados (Bevilagua et al., 2004; Rocha et al., 2004), aunque las eficiencias aún no son altas, para lo cual habría necesidad de mantener en solución el cromo en estado +6 .

Aunque en nuestro trabajo no se lograron encontrar buenos solventes y las mejores condiciones para extraer selectivamente el cromo mediante el proceso de extracción líquido-líquido, es importante seguir investigando esta posibilidad por ser una alternativa que podría tener muchas ventajas con respecto a la adsorción.

- Una técnica interesante a investigar sería la separación de los metales cuestionados, por medio de membranas a escala nanométrica o la separación con membranas líquidas.

\section{Agradecimientos}

El autor agradece la financiación de este proyecto por parte de COLCIENCIAS (proyecto 1106-1312569) y la Universidad del Valle (proyecto CI 22 408). Igualmente, agradece la colaboración administrativa y técnica dada por el Ingeniero Carlos Gómez, la ayuda de la tecnóloga Gloria Lasso en las determinaciones analíticas y el apoyo dado por todos los monitores en la realización de los ensayos.

\section{Referencias bibliográficas}

Bevilagua, T., João, J. V., \& Machado, V. G. (2004). Uso de bagaço de cana como adsorvente para a remoção de crõmio (VI). Memorias del XXVI Congreso Latinoamericano de Química, Sección Química Ambiental, Salvador, Brasil.

Cotton. F. A., Wilkinson, G., \& Gaus, P. L. (1994). Basic Inorganic Chemistry. Third Edition. John Wiley and Sons, Inc.

De Juan, D., Meseguer, V., \& Lozano, Y. L. J. (1998a). Extracción de cromo con disolventes orgánicos. Parte I. Extracción con DEHPA. Revista de Metalurgia 34(3), 253-260.

De Juan, D., Meseguer, V., \& Lozano, Y. L. J. (1998b). Extracción de cromo con disolventes orgánicos. Parte II. Extracción con TBP. Revista de Metalurgia 34 (4), 319-327.

Gould, J. P., Masingale, M. Y., \& Miller, M. (1984). Recovery of silver and mercury from COD samples by iron cementation. Journal Water Pollution Control Federation 56 (3), 280-286.

Jenkins, D., \& Snoeyink, V. L. (2003). Química del agua. México: Editorial Limusa. 
Marcus, Y., \& Sengupta, A. K. (2002). Ion exchange and solvent extraction. New York: Marcel Dekker, Inc.

Marvin, U. J. (1956). Chromium Chemistry of Chromium and its Compounds. Volume 1. New York: Reinhold Publishing Corporation.

OMS (Organisation mondiale de la Santé). (2000). Directives de qualité pour l'eau de boisson. Deuxième édition. Volume 2. Constituants inorganiques et paramètres physiques. Genève: OMS.

Outokumpu. (2002). HSC Chemistry for Windows (Chemical reaction and equilibrium software with extensive thermochemical database). Version 5.11 .

Rocha, C. G., Alfaya, R. V. S., \& Alfaya, A. A. S. (2004). O uso da palha de arroz, um residuo agroindustrial, na remediação de aguas poluidas com ions metálicos. Memorias del XXVI Congreso Latinoamericano de Química, Sección Química Ambiental, Salvador, Brasil.

Wood, J. M., \& Wang, H. K. (1983). Microbial resistance to heavy metals. Environmental Science and Technology 17 (12), 582A-590A.

Yaws, C. L. (2003). Yaws' handbook of thermodynamic and physical properties of chemical compounds. Chapter 23. Knovel Corporation. 